\title{
${ }^{1} \mathrm{H}$ NMR study of internal motions and quantum rotational tunneling in $\left(\mathrm{CH}_{3}\right)_{4} \mathrm{NGeCl}_{3}$
}

\author{
K. J. Mallikarjunaiah, ${ }^{a}$ K. Jugeshwar Singh, ${ }^{b}$ K. P. Ramesh ${ }^{b}$ and R. Damle ${ }^{a *}$
}

\begin{abstract}
$\left(\mathrm{CH}_{3}\right)_{4} \mathrm{NGeCl}_{3}$ is prepared, characterized and studied using ${ }^{1} \mathrm{H}$ NMR spin lattice relaxation time and second moment to understand the internal motions and quantum rotational tunneling. Proton second moment is measured at $7 \mathrm{MHz}$ as function of temperature in the range $300-77 \mathrm{~K}$ and spin lattice relaxation time $\left(T_{1}\right)$ is measured at two Larmor frequencies, as a function of temperature in the range 270-17 K employing a homemade wide-line/pulsed NMR spectrometers. $T_{1}$ data are analyzed in two temperature regions using relevant theoretical models. The relaxation in the higher temperatures $(270-115 \mathrm{~K})$ is attributed to the hindered reorientations of symmetric groups $\left(\mathrm{CH}_{3}\right.$ and $\left.\left(\mathrm{CH}_{3}\right)_{4} \mathrm{~N}\right)$. Broad asymmetric $T_{1}$ minima observed below $115 \mathrm{~K}$ down to $17 \mathrm{~K}$ are attributed to quantum rotational tunneling of the inequivalent methyl groups. Copyright (c) 2007 John Wiley \& Sons, Ltd.
\end{abstract}

Keywords: NMR; ${ }^{1} \mathrm{H}$; internal motions; spin lattice relaxation time; second moment; quantum rotational tunneling

\section{Introduction}

$\mathrm{ABX}_{3}(\mathrm{~A}=$ alkali metal and alkylammonium ion, $\mathrm{B}=$ metal atom and $\mathrm{X}=\mathrm{Cl}, \mathrm{Br}$ and I) systems are an interesting group of compounds because of the changes in their physical properties due to structural phase transitions. Perovskite halides $A B X_{3}$ of group IV elements such as $\mathrm{Ge}, \mathrm{Sn}$, and $\mathrm{Pb}$ have been studied extensively, partly because of their successive phase transitions and partly because of the diversity in their electrical properties such as ferroelectricity, and ionic and electronic conductivity. ${ }^{[1-3]}$ Successive replacement of hydrogen by $\mathrm{CH}_{3}$ groups in ammonium ion according to the formula $\mathrm{Me}_{x} \mathrm{NH}_{4-x}(x=1 \ldots 4)$ leads to a cation with a larger ionic radius, which may result in strong phase transitions due to changes in symmetry as well as tolerance factor. ${ }^{[4]}$ In addition to the deviation from spherical symmetry, additional symmetry elements may lead to increased reorientation motions. ${ }^{[5]}$ In alkylammonium trichlorogermanate (II) compounds, mobile chloride ion is found to be responsible for ionic conductivity and its abrupt change at the phase transitions. ${ }^{[6,7]}$

Möller and Felsche. ${ }^{[8]}$ have shown that at room temperature $(293 \mathrm{~K}),\left(\mathrm{CH}_{3}\right)_{4} \mathrm{NGeCl}_{3}$, here after abbreviated as TMAC-germanate, crystallizes in an orthorhombic modification (Pna2 1 ) with $a=$ $13.096 \AA, b=8.911 \AA, c=9.128 \AA$ and $z=4$. The TMACgermanate has been reported ${ }^{[9]}$ to reveal five phases in the temperature region $463-130 \mathrm{~K}$, in which highest temperature phase being cubic perovskite structure above $424 \mathrm{~K}$. Further, to understand the mechanism of phase transitions, the compound has been studied using alternating current (AC) conductivity, temperature dependent single crystal XRD and ${ }^{35} \mathrm{Cl} N Q R / N M R$ techniques. ${ }^{[7]}$ The title compound was also investigated by Winkler et al. ${ }^{[10]}$ using quasielastic and inelastic neutron scattering and Raman spectroscopic techniques and they observed the molecular reorientation of the whole tetramethylammonium (TMA) and methyl groups in the temperature region 446-4 K. Further, they have also observed that the structural phase transitions at 170 , 200 and $424 \mathrm{~K}$ are unrelated to the temperature-induced changes of the molecular dynamics. However, to best of our knowledge ${ }^{1} \mathrm{H}$ NMR studies as a function of temperatures have not been reported.
This motivated us to invoke low temperature (LT) dynamics in the present compound, as it is expected to be a potential candidate to do so, because diammonium hexafluorogermanate has shown quantum rotational tunneling at lower temperatures. ${ }^{[11]}$

In this article, we report the results of ${ }^{1} \mathrm{H}$ NMR relaxation time and second moment studies on the TMAC-germanate and the results are analyzed using appropriate theoretical models pertaining to reorientations of the $\left(\mathrm{CH}_{3}\right)_{4} \mathrm{~N}$ and $\mathrm{CH}_{3}$ groups at higher temperatures ( $\mathrm{HTs})$, along with quantum rotational tunneling at LTs.

\section{Experimental}

TMAC-germanate is prepared following the method described by Poskozim and Stone. ${ }^{[12]}$ All the chemicals used in this preparation are obtained from Aldrich Chem. Co. To begin with, germanium tetrachloride is prepared by the reaction of germanium oxide with hydrogen chloride, which is further reduced to $\mathrm{GeCl}_{2}$ with hypophosphorous acid $\mathrm{H}_{3} \mathrm{PO}_{2}{ }^{[13]}$ The TMAC-germanate is prepared by the addition of equal molar quantities of TMA chloride to $\mathrm{GeCl}_{2}-\mathrm{H}_{3} \mathrm{PO}_{2}$ media. ${ }^{[12]}$ The reaction can be represented as

$$
\begin{aligned}
& \mathrm{GeO}_{2}+\mathrm{H}_{3} \mathrm{PO}_{2}+2 \mathrm{HCl}+\left(\mathrm{CH}_{3}\right)_{4} \mathrm{NCl} \longrightarrow \\
& \left(\mathrm{CH}_{3}\right)_{4} \mathrm{NGeCl}_{3}+\mathrm{HPO}_{2}+2 \mathrm{H}_{2} \mathrm{O}
\end{aligned}
$$

The resulting colorless precipitate is filtered and dried under vacuum for a few days because of its hygroscopic nature. The sample is characterized by using powder XRD along with DSC (heat

\footnotetext{
* Correspondence to: R. Damle, Department of Physics, Bangalore University, Bangalore- 560 056, India.E-mail:damleraju@yahoo.com

a Department of Physics, Bangalore University, Bangalore - 560 056, India

b Department of Physics, Indian Institute of Science, Bangalore - 560 012, India
} 
flow) and the results are in good agreement with those reported earlier. ${ }^{[8]}$ The compound is finely powdered and vacuum-sealed into glass ampoules of $5 \mathrm{~mm}$ diameter in helium atmosphere for NMR studies.

${ }^{1} \mathrm{H}$ NMR second moment $\left(M_{2}\right)$ measurements are carried out, as a function of temperature in the temperature range $300-77 \mathrm{~K}$, at $7 \mathrm{MHz}$ using a homemade wide-line NMR spectrometer described elsewhere. ${ }^{[14]}$ The temperature of the sample is controlled using homemade gas flow cryostat and the temperature at the sample is measured using a Pt 100 sensor. Second moment is calculated from the derivative of the absorption signal recorded using lock-in detection.

${ }^{1} \mathrm{H}$ NMR spin lattice relaxation time $\left(T_{1}\right)$ measurements are carried out, as a function of temperature, at two Larmor frequencies $26(270-17 \mathrm{~K})$ and $11.4 \mathrm{MHz}(270-115 \mathrm{~K})$ using the homemade pulsed NMR spectrometer described earlier. ${ }^{[15]}$ Inversion recovery pulse sequence is used for the measurement of $T_{1}$. The temperature of the sample is varied from 300 to $17 \mathrm{~K}$ with the help of a continuous gas flow helium cryostat (CF200 Oxford Instruments Cryosystem) and controlled to an accuracy of $\pm 0.5 \mathrm{~K}$ with the aid of ITC-503 (Oxford instruments Intelligent Temperature Controller). Temperature of the sample is measured using a $\mathrm{Pt} 100$ sensor for temperatures as low as about $75 \mathrm{~K}$ and below which a Germanium Sensor is used. The magnetization recovery is found to be exponential within the experimental error throughout the temperature region studied.

\section{Results and Discussion}

\section{Second moment}

The second moment determined as a function of temperature in the range $300-77 \mathrm{~K}$ is shown in Fig. 1 . The second moment value remains almost constant of about $2.8(0.2) \mathrm{G}^{2}$ in the temperature range $300-160 \mathrm{~K}$ within the experimental error. Below $160 \mathrm{~K}$, the signal starts broadening and second moment increases monotonically to $24.8(0.5) \mathrm{G}^{2}$ at $114 \mathrm{~K}$. The lowest temperature that can be reached with our existing LT liquid nitrogen gas flow cryostat is $114 \mathrm{~K}$ and hence the second moment

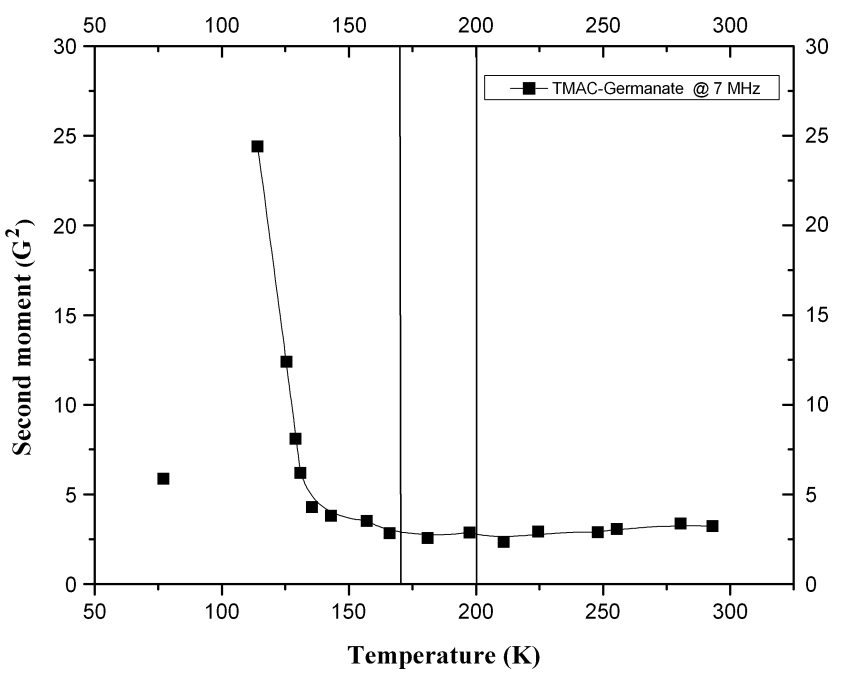

Figure 1. ${ }^{1} \mathrm{H}$ NMR Second moment data of TMAC-germanate at $7 \mathrm{MHz}$ in the temperature region $300-77 \mathrm{~K}$; the line is a guide. Vertical lines indicate phase transition temperatures. measurements have not been made in the temperature range 114-77 K. The second moment data at $77 \mathrm{~K}$ has been obtained by directly immersing the sample tube in liquid nitrogen dewar. At $77 \mathrm{~K}$, the signal becomes narrow leading to a second moment of $5.5(0.4) \mathrm{G}^{2}$.

There are many second moment simulation studies reported in the literature, including those for TMA ion in different complexes. ${ }^{[16-23]}$ We have calculated the second moment using standard models. The rigid lattice second moment can be calculated for a rigid $\left(\mathrm{CH}_{3}\right)_{4} \mathrm{~N}$ ion using the formula ${ }^{[24]}$

$$
M_{2}=\frac{9}{10} \frac{\gamma^{2} \hbar^{2}}{r^{6}}+\frac{81}{20} \frac{\gamma^{2} \hbar^{2}}{R^{6}}+M_{H T}
$$

where $\gamma=2.675 \times 10^{4} \mathrm{G}^{-1} \mathrm{~s}^{-1}$ is the nuclear gyromagnetic ratio of protons and $\mathrm{M}_{2}$ is measured in $\mathrm{G}^{2}$. Following Albert et al., ${ }^{[25]}$ we have used $r=1.78 \AA$ as the distance between the protons belonging to the same $\mathrm{CH}_{3}$ group and $R=3.04 \AA$ as the average distance between the protons belonging to different groups. Moreover, the $A$ and $B$ values obtained from our $T_{1}$ results also support that the inter proton distances do not deviate appreciably from these values. We have chosen the high temperature $(\mathrm{HT})$ residual second moment ${ }^{[26]}$ to be $1 \mathrm{G}^{2}$. From this data, we have calculated the rigid lattice $M_{2}$ value as $28 G^{2}$ which is fairly comparable with our LT second moment value of $24.8 \mathrm{G}^{2}$ (at $114 \mathrm{~K}$ ). The observed second moment value of $24.8 \mathrm{G}^{2}$ is in good agreement with the rigid lattice second moment value for the TMA ion calculated in TMA bromide by Blears et al. ${ }^{[19]}$ and also as observed experimentally by Prabhumirashi et al. in $\left(\left(\mathrm{CH}_{3}\right)_{4} \mathrm{~N}\right)_{2} \mathrm{SnCl}_{6}{ }^{[20]}$ The estimation of activation energy ${ }^{[21]}$ from the formula $E_{\mathrm{a}}=155 T_{\mathrm{C}} \mathrm{J} / \mathrm{mol}$, where $T_{C}$ is the temperature at which the NMR spectrum narrows, yields a value of $18 \mathrm{~kJ} / \mathrm{mol}$. Since the line narrowing observed in this complex seems to occur in a single step, it can be concluded that the individual $\mathrm{C}_{3}$ reorientation of four $\mathrm{CH}_{3}$ groups in the cation and the overall reorientation of the cation as a whole occur simultaneously at a very narrow interval of temperature. Similar behavior has been observed in $\left(\mathrm{CH}_{3}\right)_{4} \mathrm{NCdCl}_{3}$, but at much lower temperatures. ${ }^{[22]}$

The observed plateau second moment of about $2.8 \mathrm{G}^{2}$ in the HT region suggests that both TMA and methyl group reorientations are active down to $160 \mathrm{~K}$ and below which, motions of both the groups start freezing. Our results show no resolved plateau in second moment, indicating that the two motions do not have widely differing correlation frequencies. ${ }^{[23]}$ The finite second moment of $5.5 \mathrm{G}^{2}$ at $77 \mathrm{~K}$ suggests the existence of rotational tunneling of the methyl groups, ${ }^{[27]}$ which is consistent with our LT $T_{1}$ results discussed below.

\section{Spin lattice relaxation time}

Figure 2 shows the variation of ${ }^{1} \mathrm{HNMR} T_{1}$ with inverse temperature $(1000 / T)$ at 26 and $11.4 \mathrm{MHz}$. $T_{1}$ measurements have been carried out in the temperature region $270-17 \mathrm{~K}$ at $26 \mathrm{MHz}$, while at 11.4 $\mathrm{MHz}$ the temperature range is restricted to $270-115 \mathrm{~K}$, due to poor signal to noise ratio at lower temperatures. The $T_{1}$ data are analyzed in two parts (a) the high temperature (HT) region $(270-115 \mathrm{~K})$ as shown in Fig. 3 and (b) the low temperature (LT) region $(115-17 \mathrm{~K})$ as shown in Fig. 4.

\section{High temperature (HT) region (270-115 K)}

Figure 2 is redrawn as Fig. 3 in a limited temperature range (270-115 K) for convenience of presentation of the fitting at both 


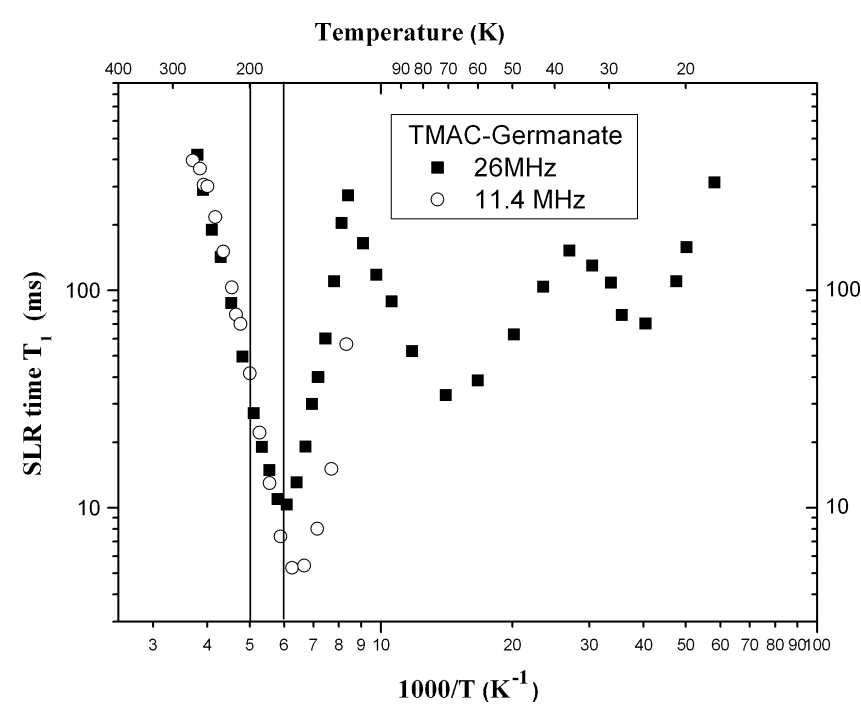

Figure 2. ${ }^{1} \mathrm{H}$ NMR $T_{1}$ data of TMAC-germanate at $26 \mathrm{MHz}$ (square) (270-17 K) and $11.4 \mathrm{MHz}$ (open circle) (270-115 K). Vertical lines indicate phase transition temperatures.

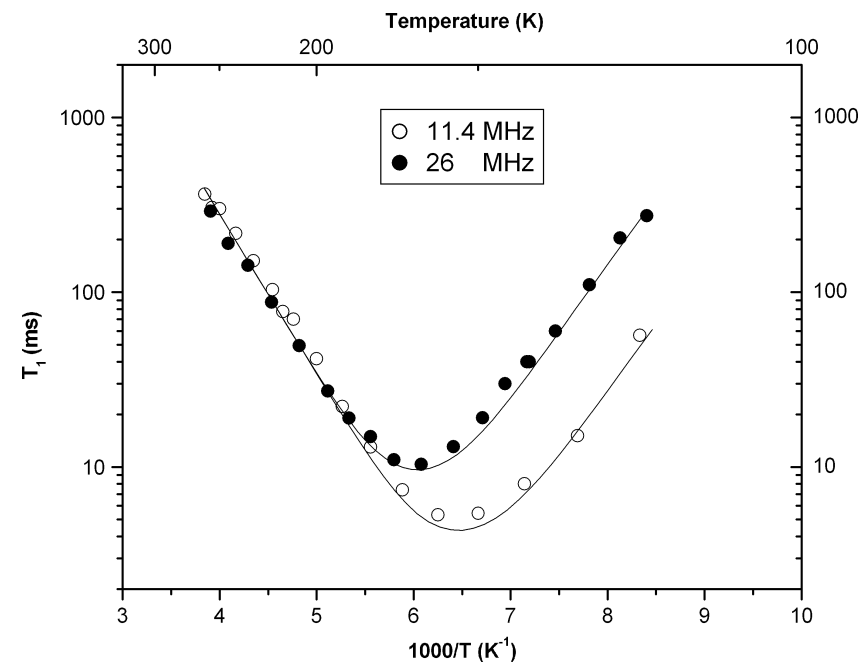

Figure 3. ${ }^{1} \mathrm{H}$ NMR $T_{1}$ data of TMAC-germanate at $26 \mathrm{MHz}$ (full circle) and $11.4 \mathrm{MHz}$ (open circle). Solid line represents the theoretical fit ${ }^{[25]}$.

frequencies. Figure 3 shows that, $T_{1}$ graphs at both frequencies show a single asymmetric minimum, instead of two minima expected, corresponding to each symmetric group (TMA and $\mathrm{CH}_{3}$ ) present in the compound as in most of the TMA compounds. However, similar behavior has been observed in several other compounds. $^{[20,30]}$

\section{Theory}

The $T_{1}$ behavior in TMA compounds can be explained using a modified Bloembergen-Purcell-Pound (BPP) approach, as given by Albert et al. ${ }^{[25]}$ Albert et al. have studied the ${ }^{1} \mathrm{H} T_{1}$ in several TMA halides and the experimental results have been analyzed by considering the TMA ion and methyl group reorientations about their $C_{3}$ axes. In TMA compounds, the two motions that mainly contribute to the relaxation are (i) random reorientation of the $\mathrm{CH}_{3}$ groups with correlation time $\tau_{\mathrm{c}}$ and (ii) isotropic tumbling of the TMA ion (whole cation) with a correlation time $\tau_{\mathrm{c} 1}$. They modulate

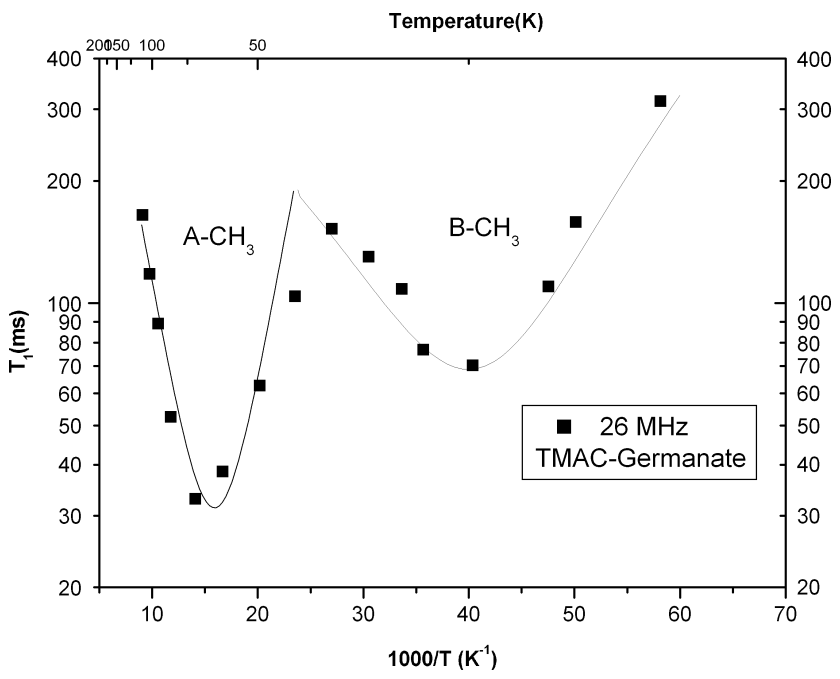

Figure 4. $T_{1}$ data of TMAC-germanate in the temperature range $115-17 \mathrm{~K}$. Solid lines represent theoretical fit ${ }^{[28,29]}$ considering inequivalent $\mathrm{CH}_{3}$ groups.

the intramethyl and intermethyl dipole-dipole interactions and facilitate the spin lattice relaxation.

Albert et al. ${ }^{[25]}$ have assumed that the observed curves could be described by two superimposed BPP curves ${ }^{[31]}$ and the effective relaxation rate is the sum of the relaxation rates due to the intramethyl and intermethyl contributions and hence can be written as

$$
T_{1}^{-1}=A f\left(\tau_{\mathrm{c} 2}\right)+B f\left(\tau_{\mathrm{c} 1}\right)
$$

where

$$
\begin{aligned}
\tau_{\mathrm{c} 2}^{-1} & =\tau_{\mathrm{c}}{ }^{-1}+\tau_{\mathrm{c} 1}{ }^{-1} \\
A & =\frac{9}{20} \frac{\gamma^{4} \hbar^{2}}{r^{6}} \\
B & =\frac{3}{20} \frac{\gamma^{4} \hbar^{2}}{r^{6}}+\frac{27}{10} \frac{\gamma^{4} \hbar^{2}}{R^{6}}
\end{aligned}
$$

in which, $\gamma=2.675 \times 10^{4} \mathrm{G}^{-1} \mathrm{~s}^{-1}$ is the nuclear gyromagnetic ratio of protons, $r$ is the inter proton distance within the methyl group and $R$ is the distance between the centers of the triangles formed by the three protons of each $\mathrm{CH}_{3}$ group. $\tau_{\mathrm{c}}, \tau_{\mathrm{c} 1}$ and $\tau_{\mathrm{c} 2}$ respectively represent the correlation time of the methyl group, TMA cation and the effective correlation time due to both methyl and TMA cation reorientations around the preferred axes. The function $f(\tau)$ is given by

$$
f(\tau)=\tau\left(1+\omega^{2} \tau^{2}\right)^{-1}+4 \tau\left(1+4 \omega^{2} \tau^{2}\right)^{-1}
$$

where ' $\tau$ ' represents the correlation time of the motion and is assumed to obey the Arrhenius equation given by

$$
\tau=\tau_{0} \exp \left(E_{\mathrm{a}} / R T\right)
$$

Here $\tau_{0}$ and $E_{\mathrm{a}}$ are called pre-exponential factor and activation energy of the corresponding motion respectively.

The $T_{1}$ data in this temperature region $(270-115 \mathrm{~K})$ have been analyzed using the Albert et al. model given by Eqn (1). Generally, one uses theoretically calculated values of $A=8.05 \times 10^{9} \mathrm{~s}^{-2}$ and $B=4.61 \times 10^{9} \mathrm{~s}^{-2}$ for TMA compounds using well-known 
Table 1. Motional parameters evaluated from Albert et al. model for TMAC-germanate in the temperature region ( $270-115 \mathrm{~K})$. The values given in the parentheses represent the errors

\begin{tabular}{|c|c|c|c|c|c|c|}
\hline \multirow[b]{2}{*}{$\begin{array}{l}\text { Frequency } \\
\mathrm{MHz}\end{array}$} & \multicolumn{2}{|c|}{ Methyl } & \multicolumn{2}{|c|}{ TMA } & \multirow[b]{2}{*}{$A 10^{9} \mathrm{~s}^{-2}$} & \multirow[b]{2}{*}{ B $10^{9} \mathrm{~s}^{-2}$} \\
\hline & $\begin{array}{c}E_{a} \\
\mathrm{~kJ} / \mathrm{mol}\end{array}$ & $\tau_{c o} 10^{-13} \mathrm{~s}$ & $\begin{array}{c}E_{a} \\
\mathrm{~kJ} / \mathrm{mol}\end{array}$ & $\tau_{c o} 10^{-13} \mathrm{~s}$ & & \\
\hline 11.4 & $13.59(0.7)$ & $2.75(0.4)$ & $18.35(0.8)$ & $0.1(0.02)$ & $8.10(1.2)$ & $4.65(0.9)$ \\
\hline 26 & $13.30(0.5)$ & $2.90(0.4)$ & $18.76(0.5)$ & $0.1(0.02)$ & $8.02(1.0)$ & $4.50(0.7)$ \\
\hline
\end{tabular}

bond length and bond angles of the TMA molecule, as estimated by Albert et al. We have fit the Albert et al. model as given by Eqn (1) to our experimental data by considering $A$ and $B$ also as fit parameters, independently for both the frequencies and the theoretical fit curves are shown as solid lines in Fig. 3. The obtained $A$ and $B$ values are in good agreement with the calculated values. The fit parameters (activation energies and pre-exponential factors) obtained for both the frequencies are within the error limits (shown in the parentheses) and are given in Table 1.

The observed motional parameters are found to be in good agreement with those reported for other TMA compounds. ${ }^{[20,21,25]}$ On comparing the activation energies of both the TMA and methyl groups in $\left(\mathrm{CH}_{3}\right)_{4} \mathrm{NMCl}_{3}(\mathrm{M}=\mathrm{Cd}$, $\mathrm{Sn}$ and $\mathrm{Ge})$ salts, it can be noticed that activation energy of the TMA/methyl groups increases with decreasing metal ion radius. ${ }^{[21,32]}$ This can be correlated to the increased volume (higher metal ion radius) available for the motion of the symmetric groups.

\section{Low temperature (LT) range (115-17 K)}

The effects of rotational tunneling of $\mathrm{CH}_{3}$ groups on proton spin lattice relaxation have been discussed by several authors. ${ }^{[33-43]}$ Nonexponential magnetization recovery and multiple minima in $T_{1}$ graphs at LTs are some of the signatures of the tunneling. Tunneling motions influence the proton spin lattice relaxation in various striking ways and no complete understanding of the spin-phonon interaction has been reached. ${ }^{[25]}$ The $T_{1}$ results in the title compound show two broad asymmetric minima in the temperature region $115-17 \mathrm{~K}$ and the magnetization recovery is found to be exponential throughout the temperature region studied. Here we have tried to fit our $T_{1}$ results with the model proposed by Koksal et al. ${ }^{[28]}$ by considering inequivalent $\mathrm{CH}_{3}$ groups ( $A$ type and $B$ type). Similar observation of multiple minima have been reported. ${ }^{[29,42,44]}$

\section{Theory}

Koksal et al. have studied a number of acetates by ${ }^{1} \mathrm{H}$ NMR $T_{1}$ measurements and explained their experimental results by considering the temperature dependence of tunnel splitting. At LTs, it is assumed that only the torsional ground state $(n=0)$ of the methyl group is occupied and thermal excitations to the first level $(n=1)$ can be described by the correlation time

$$
\tau_{c}^{T}=\tau_{\text {co }}^{\top} \exp \left(E_{01} / R T\right)
$$

where $E_{01}$ is the energy difference between the ground and first excited states of the torsional oscillator levels. The relaxation occurs when spin-flips are connected with the tunnel splittings. When the tunneling frequency is larger than the Larmor frequency, one can write the expression for the relaxation rate by considering only the intramethyl interaction and temperature dependence of the tunnel frequency, as

$$
\frac{1}{T_{A E}}=C_{A E} \sum_{m=-2}^{2} \frac{m^{2} \tau_{c}^{T}}{1+<\varpi_{T}>^{2}\left(\tau_{c}^{T}\right)^{2}}
$$

where $<\varpi_{T}>^{2}$ is the average tunnel frequency ${ }^{[45]}$ and is given by

$$
<\varpi_{T}>=\frac{\varpi_{T}^{0}-\varpi_{T}^{1} \exp \left(-E_{01} / R T\right)}{1+\exp \left(-E_{01} / R T\right)}
$$

and

$$
C_{A E}=\frac{9}{20} \frac{\gamma^{4} \hbar^{2}}{r^{6}} d_{0}^{2}
$$

In the above equations, $\omega_{T}{ }^{0}$ and $\omega_{T}{ }^{1}$ are the ground and first excited torsional tunnel splittings; $d_{0}$ is the matrix element of the space part of the dipolar operator between the ground state wave functions of the harmonic oscillator. ${ }^{[29]}$

We have fit the $T_{1}$ data between 115 and $17 \mathrm{~K}$ to the model used by Koksal et al. as shown in Fig. 4. The best fit parameters are given in the Table 2 . The motional parameters are comparable with those reported for $\mathrm{Cd}\left(\mathrm{CH}_{3} \mathrm{COO}\right)_{2}{ }^{[29]}$ except the activation energy, which is comparatively less, but in reasonable agreement with the results of Senthil Kumaran et al. ${ }^{[32]}$

\section{Conclusions}

The TMAC-germanate exhibits thermally activated reorientations of the TMA and methyl groups. The occurrence of single asymmetric minimum might be due to the approximately same reorientation rate of both the symmetric groups in the compound. There is no perceptible change in the $T_{1}$ behavior at the reported phase transition temperatures. The environment of TMA and methyl groups does not seem to have caused any effect to reveal

\begin{tabular}{|c|c|c|}
\hline \multirow[b]{2}{*}{ Parameters } & \multicolumn{2}{|c|}{ Best fit values } \\
\hline & $A$ type & $B$ type \\
\hline$E_{01}(\mathrm{~kJ} / \mathrm{mol})$ & $0.67(0.01)$ & $0.092(0.01)$ \\
\hline$\tau_{c 0}^{\top}(\mathrm{s})$ & $0.09(0.05) \times 10^{-11}$ & $4.5(0.05) \times 10^{-11}$ \\
\hline$C_{\mathrm{AE}}\left(\mathrm{s}^{-2}\right)$ & $40 \times 10^{9}$ & $6 \times 10^{9}$ \\
\hline$\omega^{0}{ }_{T}\left(\mathrm{~s}^{-1}\right)$ & $6.9 \times 10^{9}$ & $9 \times 10^{9}$ \\
\hline$\omega^{1} T\left(s^{-1}\right)$ & $100 \times 10^{9}$ & $45 \times 10^{9}$ \\
\hline
\end{tabular}
any perceptible changes in our NMR data. At lower temperatures, $T_{1}$ results suggest the existence of the inequivalent methyl groups undergoing quantum rotational tunneling. 


\section{Acknowledgements}

Our research is supported by grants from Department of Atomic Energy (DAE-BRNS) and University Grants Commission, Government of India.

\section{References}

[1] Yamada K, Mikawa K, Okuda T, Knight KS. J. Chem. Soc., Dalton Trans. $2002 ; 2112$.

[2] Okuda T, Gotou S, Takahashim T, Terao H, Yamada K. Z. Naturforsch. 1996; 51a: 686.

[3] Okuda T, Chowdari BVR. In Proceedings of the $6^{\text {th }}$ Asian Conference on Solid State Ionics: Science and Technology. World Scientific: Singapore, 1998; 335

[4] Rao CNR, Rao KJ. Phase Transitions in Solids. Mcgraw-Hill: New York, 1978.

[5] Serr BR, Heckert G, Rotter HW, Thiele G, Ebling D. J. Mol. Struct. 1995; 348: 95.

[6] Yamada K, Isobe K, Tsuyama E, Okuda T, Furukawa Y. Solid State Ionics. 1995; 79: 152.

[7] Yamada K, Isobe K, Okuda T, Furukawa Y. Z. Naturforsch. 1994; 49a: 258.

[8] Möller A, Felsche J. J. Appl. Crystallogr. 1979; 12: 617.

[9] Fütterer K, Depmeier W, Petŕ̌ček V. Acta Crystallogr. 1995; B51: 768.

[10] Winkler B, Kaiser I, Chall M, Coddens G, Hennion B, Kahn R. Physica B 1997; 234-236: 70.

[11] Svare I, Ain el Hiah Abd el Haleem. Phys. Scr. 1979; 19(4): 351.

[12] Poskozim PS, Stone AL. J. Inorg. Nucl. Chem. 1970; 32: 1391.

[13] Poskozim PS, Stone AL. J. Organomet. Chem. 1969; 16: 314.

[14] Mallikarjunaiah KJ, Damle R. Vignana Bharathi 2005; 17(1): 50 .

[15] Kannan R, Ramesh KP, Ramakrishna J. Phys. Status Solidi b. 2001; 225(1): 157.

[16] Goc R. J. Magn. Reson. 1998; $132: 78$.

[17] Goc R. Comput. Phys. Comm. 2004; 162: 102.

[18] Goc R.Z. Naturforsch. 2003; 58a: 537.

[19] Blears DJ, Danyluk SS, Bock E. J. Phys. Chem. 1968; 72(6): 2269.

[20] Prabhumirashi LS, Ikeda R, Nakamura D. Ber. Bunsenges. Phys. Chem. 1981; 85: 1142.
[21] Waugh JS, Fedin El. Soviet Phys. Solid State. 1963; 4: 1633.

[22] Tsang T, Utton DB. J. Chem. Phys. 1976; 64(9): 3780.

[23] Andrew ER, Canepa PC. J. Magn. Reson. 1972; 7: 429.

[24] Abragam A. The Principles of Nuclear Magnetism. Oxford University Press: New York, 1961

[25] Albert S, Gutowsky HS, Ripmeester JA. J. Chem. Phys. 1972; 56(7): 3672.

[26] Mahajan M, Nageswara Rao BD. J. Phys. C: Solid State Phys. 1974; 7: 995.

[27] Ramesh KP, Devaraj N, Ramakrishna J, Venu K, Sastry VSS. Spectrochim. Acta 1993; 49A: 1773.

[28] Köksal F, Rössler E, Sillescu H. J. Phys. C: Solid State Phys. 1982; 15: 5821.

[29] Sobol WT, Sreedharan KR, Cameron IG, Pintar MM. Z. Naturforsch. 1985; 40a: 1075.

[30] Mallikarjunaiah KJ, Paramita KC, Ramesh KP, Damle R. Solid State Nucl. Magn. Reson. 2007; 32(1): 11.

[31] Bloembergen N, Purcell EM, Pound RV. Phys. Rev. 1948; 73(7): 679.

[32] Senthil Kumaran S, Ramesh KP, Ramakrishna J. Mol. Phys. 2001; 99(16): 1373.

[33] Latonowicz L. J. Concepts Magn. Reson. A 2005; 27(1): 38

[34] Latonowicz L. J. Phys. Chem. A 2004; 108(51): 11172.

[35] Latonowicz L, Medycki W, Jakubas R. J. Phys. Chem. A 2005; 109(14): 3097.

[36] Horsewill AJ. Prog. Nucl. Magn. Reson. Spectrosc. 1999; 35: 359.

[37] Svare I, Raaen AM, Finland WO. Physica B 1985; 128: 144.

[38] Svare I, Raaen AM, Thorkildsen G. J. Phys. C: Solid State Phys. 1978; 11: 4069.

[39] Ylinel EE, Tuohi JE, Niemela LKE. Chem. Phys. Lett. 1974; 24: 447.

[40] Clough S, Horsewill AJ, Johnson MR, Sutchliffe JH, Tomsah IBI. Mol. Phys. 1994; 81: 975.

[41] Ingman LP, Punkkinen M, Vuorimaki AH, YLinen EE. J. Phys. C: Solid State Phys. 1985; 18: 5033.

[42] Tuohi JE, Ylinen EE. Phys. Scr. 1976; 13: 253.

[43] Ingman LP, Punkkinen M, Ylinen EE, Dimitropoulos C. Chem. Phys. Lett. 1986; 125: 170.

[44] Mallikarjunaiah KJ, Ramesh KP, Damle R. Phys. Status Solidi b 2007; 244(10): 3809.

[45] Müller -Warmuth W, Schüler R, Prager M, Kollmar A. J. Chem. Phys. 1978; 69(6): 2382. 